\title{
IUFOST2006/616 Hollow fibre membrane contactor: new technology to control mass transfers in liquid food separations
}

\author{
I. Souchon ${ }^{\mathrm{a}}$, F. Gascons Viladomat ${ }^{\mathrm{b}}, \mathrm{M}$. Bes $^{\mathrm{c}}, \mathrm{V}$. Athès ${ }^{\mathrm{a}}$ and M. Marin ${ }^{\mathrm{a}}$ \\ ${ }^{a}$ INRA - INA GP - Joint Research Unit : Food Process Engineering \& Microbiology, 78850 Thiverval \\ Grignon, France \\ baboratoire de Génie chimique UMR CNRS-UPS 5503, 118 route de Narbonne, 31062 Toulouse Cedex, \\ France \\ ${ }^{\mathrm{c}}$ INRA, Unité Expérimentale de Pech Rouge, 11430 Gruissan, France \\ souchon@grignon.inra.fr
}

\begin{abstract}
Membrane contactors are proposed as a new technology to control mass transfer of volatile compounds in separation processes. This technology consists in the use of a porous membrane which permits to immobilise the interface of transfer. Applications presented in this study concern the partial dealcoholisation of wine and the aroma recovery from agro-industrial processing waters. For these two applications, the stake is the selective transfer of the volatile compounds: 1) to maximise the ethanol transfer and minimise the aroma compounds in the case of partial dealcoholisation of wine and 2) to control the selective extraction of odorous compounds in the case of the deodorisation treatment of agro-industrial processing waters. Using the same technology, a hollow fibre membrane contactor, different configurations have been studied: Membrane-based solvent extraction (MBSE), Membrane Air-Stripping (MAS) and Isothermal Membrane Distillation (IMD). Based on systematic experimental studies on synthetic aqueous medium containing fifteen aroma compounds with a large range of physico-chemical properties an analysis of mass transfer in each configuration has been performed. The relation between mass transfer coefficients and partition coefficients in MAS and MBSE has been established (figure 1). Limiting steps of mass transfer have been identified and a resistance-inseries model to predict mass transfer has been proposed. The feasibility of a partial dealcoholisation of wine using hollow fibre membrane contactors has been studied according to the isothermal membrane distillation (IMD) principle. The selective transfer between ethanol and volatile aroma compounds has been analyzed according to thermodynamic properties of a set of key wine aroma compounds. The global analysis of the three configurations (MBSE, MAS and IMD) also allowed identifying which process is the most adequate regarding the nature of the volatile compounds involved.

Figure 1. Relation between the overall aqueous phase-based mass transfer coefficient (Kf, m.s-1) of volatile compounds and their partition coefficient in MAS (left side) and MBSE (right side). KEYWORDS. Volatile compounds, odorous effluent, wine dealcoholisation, membrane process
\end{abstract}

